\title{
FERTILITY MODELLING
}

\author{
MARE VÄHI \\ Institute of Mathematics and Statistics, University of Tartu, Tartu, Estonia
}

\begin{abstract}
In order to describe age-specific fertility curves, different theoretical approaches are employed. By modelling a time-variant fertility curve, it is possible to forecast reproductive behaviour of Estonian women in the future. The approach provided here is a novel way to forecast fertility.
\end{abstract}

Keywords: age-specific fertility rate; beta distribution; forecast.

For years, modelling of fertility curves by theoretical distribution has been used to describe reproductive patterns of women in child-bearing age. Several mathematical models fit adequately with fertility curves. The curve-fitting approach is based on the age-specific fertility rates.

The age-specific fertility rates are calculated on the basis of the following data: the number of women in child-bearing age (aged 15-49) and the number of children born by these women.

Using $f_{x}$ to designate the number of women aged $\mathrm{x}$ years $(\mathrm{x}=15,16, \ldots 49)$ and $b_{x}$ to designate the number of children born within a year by women aged $\mathrm{x}$, the fertility rate for the women aged $\mathrm{x}$ may be calculated using the following equation:

$$
v_{x}=b_{x} / f_{x} .
$$

To provide a more explicit interpretation, the fertility rate is often expressed as the calculated value multiplied by 1,000 , and the result represents then the number of children born within a year for 1,000 women.

The new approach to stochastic forecast of the age-specific fertility rates can be summarized as follows. 
(1) Fit the suitable family of distributions for modelling the fertility rates.

(2) Estimate the parameters of distribution.

(3) Forecast the parameters using the time series model.

(4) Use the forecast parameters to forecast the model for age-specific fertility rates by one-year age groups.

The methodology was applied to Estonian fertility data. Empirical data were taken from the Statistical Database of Statistics Estonia.

The model was designed based on the data for years between 1991 and 2009. Figure 1 shows the variation of respective fertility rates.

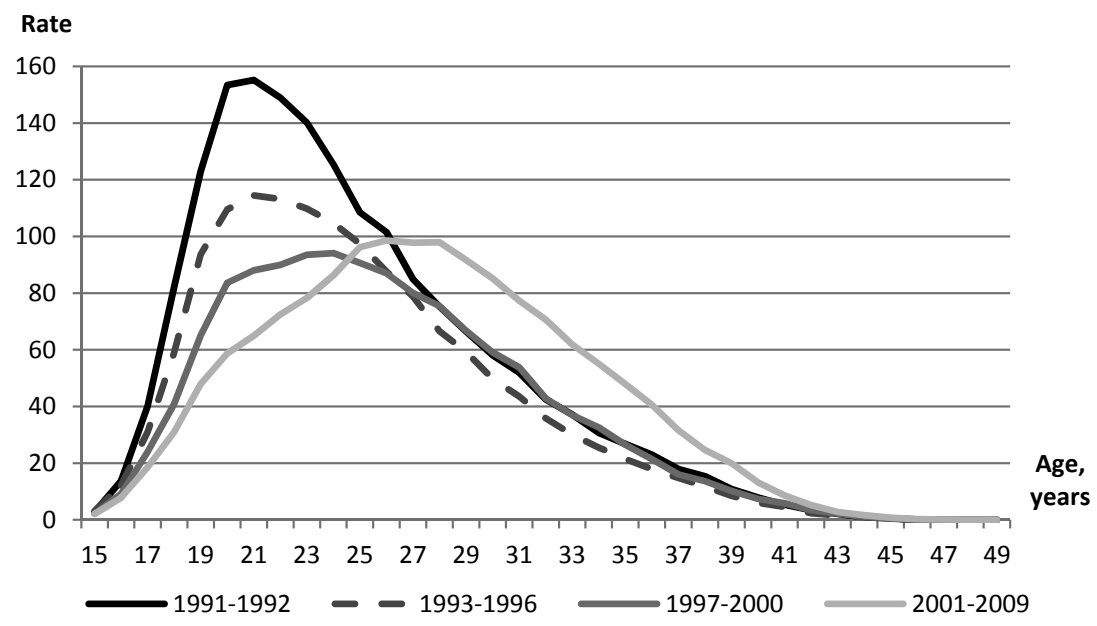

Figure 1. Age-specific fertility rate

In the above-mentioned range of years, four periods may be distinguished: years 1991-1992, 1993-1996, 1997-2000 and 2001-2009. In the first period, fertility rates are high in all age groups and the highest in the group of women aged 20 to 22. In the second period (1993-1996), the delay in giving births may be observed. The number of young mothers (aged 20-24) falls sharply, but the number of mothers in the age group 25-36 also decreases.

In the period of 1997-2000, the number of births still decreases in the age group 20-24, but the fall is not as sharp as in the earlier period. In the older groups (starting from the age of 25 years), the number of births remains on the level of 1993. An additional trend, however, can be noticed - the number of births increases in the group of women aged 31 and 32. 
Compared with the year 1996, the number of mothers has decreased even more by the year 2000 in younger age groups, whereas it has increased in older age groups, which suggests that the women who delayed child births in the earlier years started to give birth. Consequently, the number of mothers has grown in the age group 27-38.

In the last period or in this century, the number of mothers has further declined in the age group 18-24, but the increase in the number of births has been considerable in the age group 25-42.

In addition, the curves that were inclined to the left in the first periods are becoming more and more symmetrical. A symmetrical, bell-shaped fertility curve is characteristic of the fertility curves of several European countries.

As shown in Figure 1, the fertility curves of the studied periods differ considerably.

The next step is to find theoretical distribution so as to describe the fertility curves. Keeping in mind the aim of using a distribution appropriate for describing fertility curves to forecast the fertility, it would be beneficial to describe all the curves within the observed period by means of the same type of distribution.

The researchers studying the fertility curves of different countries have used various distributions for approximation. The most widely used distributions are the gamma distribution, the beta distribution and the Hadwiger distribution. The latter has been employed, for example, in Sweden, Denmark, France and Austria. The beta distribution has been used in various European countries, whereas a mixture of distributions has been used in Ireland and Great Britain. By using the said distributions, we also tried to approximate the Estonian data.

The gamma density function is expressed as follows:

$$
f(x)=\frac{x^{\gamma-1} \exp (-x / \beta)}{\beta^{\gamma} \Gamma(\gamma)},
$$

wherein $\beta$ and $\gamma$ represent the distribution parameters and $\Gamma(\gamma)$ is the gamma function.

Hadwiger function is expressed as

$$
h(x)=\frac{\alpha \beta}{\gamma \sqrt{\pi}}\left(\frac{\gamma}{x}\right)^{3 / 2} \exp \left\{-\beta^{2}\left(\frac{\gamma}{x}+\frac{x}{\gamma}-2\right)\right\},
$$


wherein $\alpha, \beta$ and $\gamma$ represent estimated parameters and $\mathrm{x}$ is the age of the mother at childbirth.

The following interpretation of the parameters has been suggested: the parameter $\alpha$ is associated with the general fertility rate, the parameter $\beta$ determines the height of the curve, and $\gamma$ is associated with the average age of mothers (Chandola et al. 1999).

The density function for beta distribution is expressed as

$$
f(x)=\frac{x^{\alpha-1}(1-x)^{\beta-1}}{B(\alpha, \beta)},
$$

wherein $\alpha$ and $\beta$ represent distribution parameters and $B(\alpha, \beta)$ is the beta function.

Neither the gamma distribution nor the Hadwiger distribution gave any adequate approximation for the observed years (Figures 2 and 3).

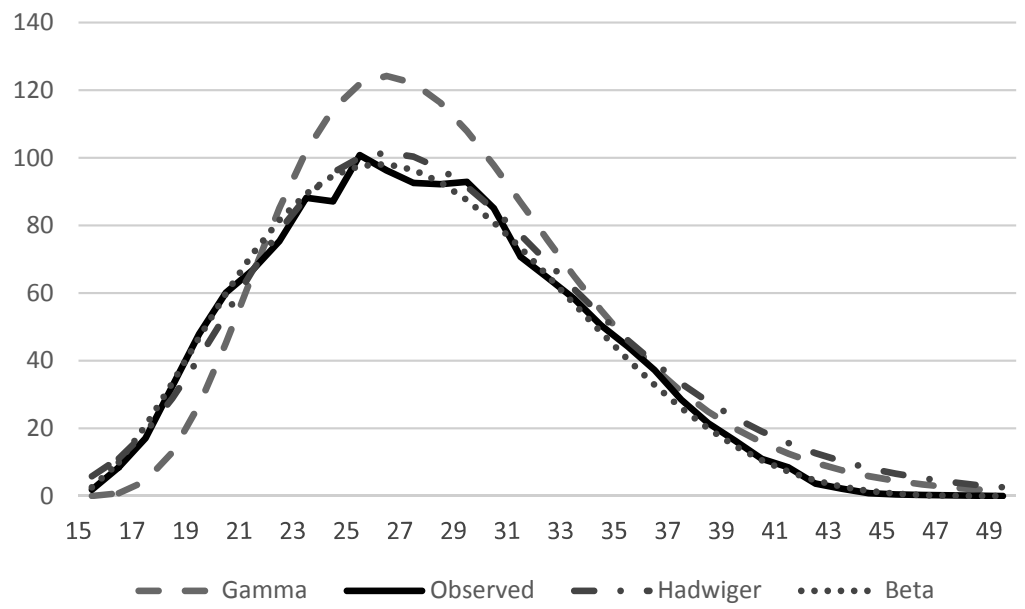

Figure 2. Age-specific fertility rates, 2008.

The distribution that fitted best for describing the curves under observation was the beta distribution. 


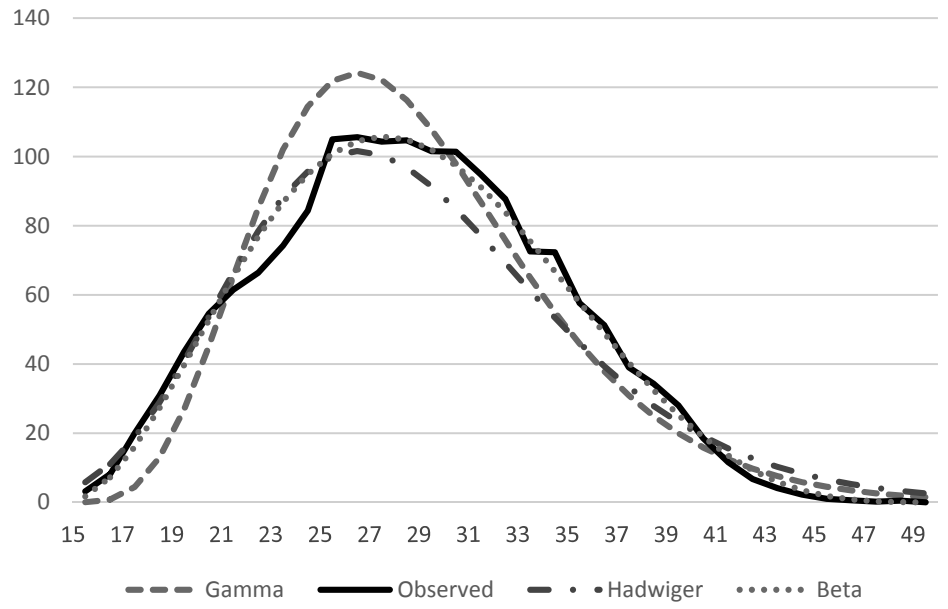

Figure 3. Age-specific fertility rates, 2008.

As the next step, the parameters of the selected distribution are assessed for the fertility curves all of the observed years.

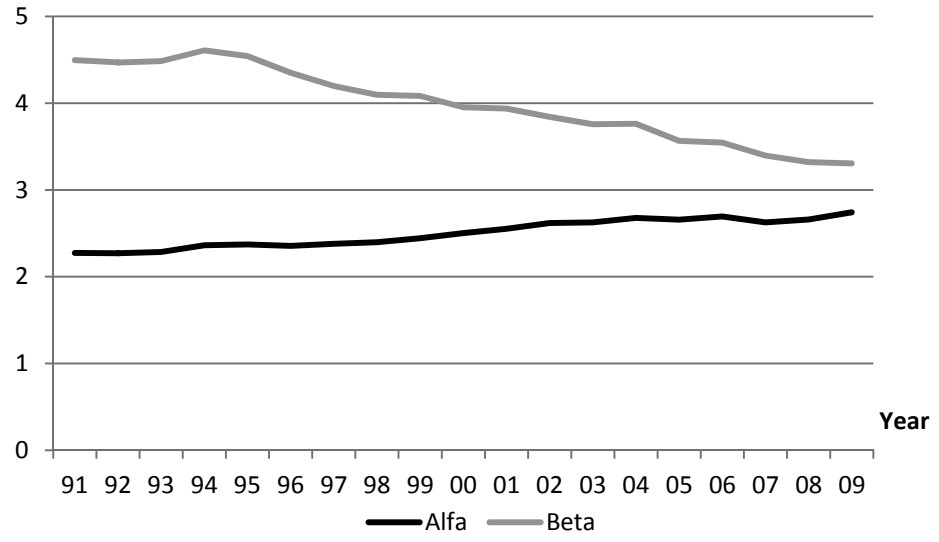

Figure 4. Dynamics of distribution parameters.

The change in the distribution parameters $\alpha$ and $\beta$ is substantially linear. The value of the first parameter is growing (Figure 4), the value of the parameter $\beta$ decreases. 
So as to use the results obtained by fitting the distributions to forecast the fertility in the following years, the possible future values of the parameters are assessed. The results are shown in Figures 5 and 6.

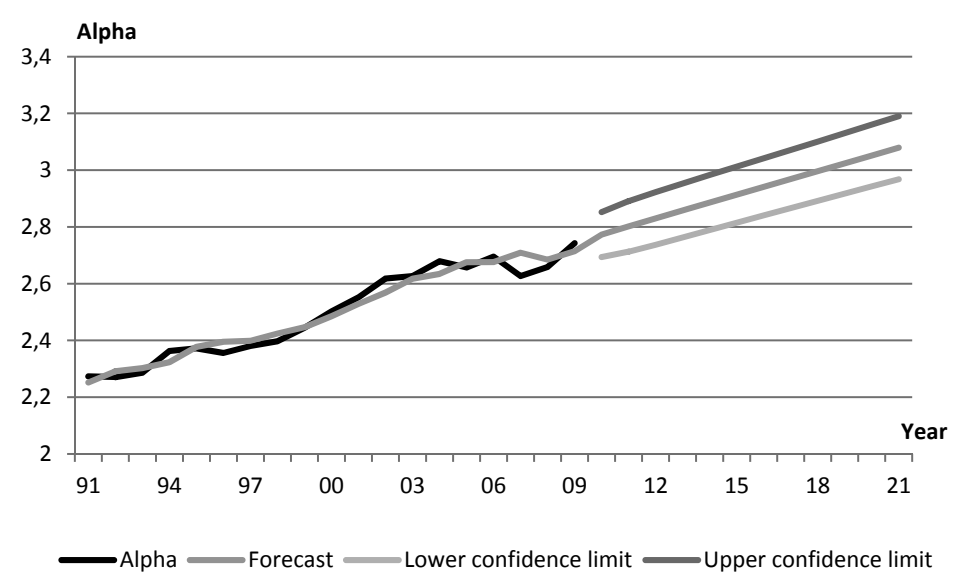

Figure 5. The change of parameter alpha.

After that, the probable future values of the parameters for the following periods are determined. The values of the parameters and the predicted values with their confidence level (confidence level 0.95) are shown in Figures 5 and 6 . The linear trend, of course, calls for caution when making long-term predictions. The said change will probably not last long.

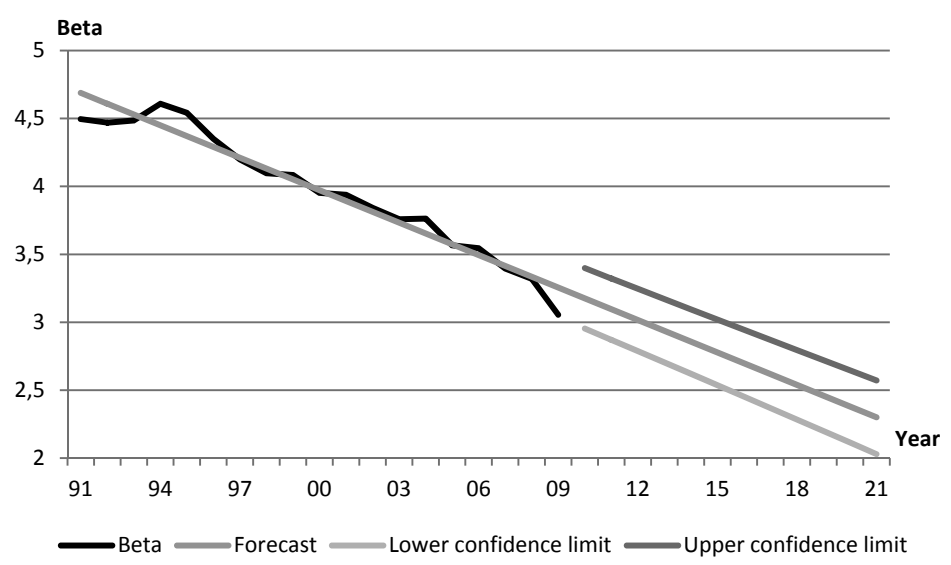

Figure 6. The change of parameter beta. 
By using the values of the parameters, the fertility curves for the coming years can be modelled. The fertility curve modelled for the year 2021 is been provided in Figure 7.

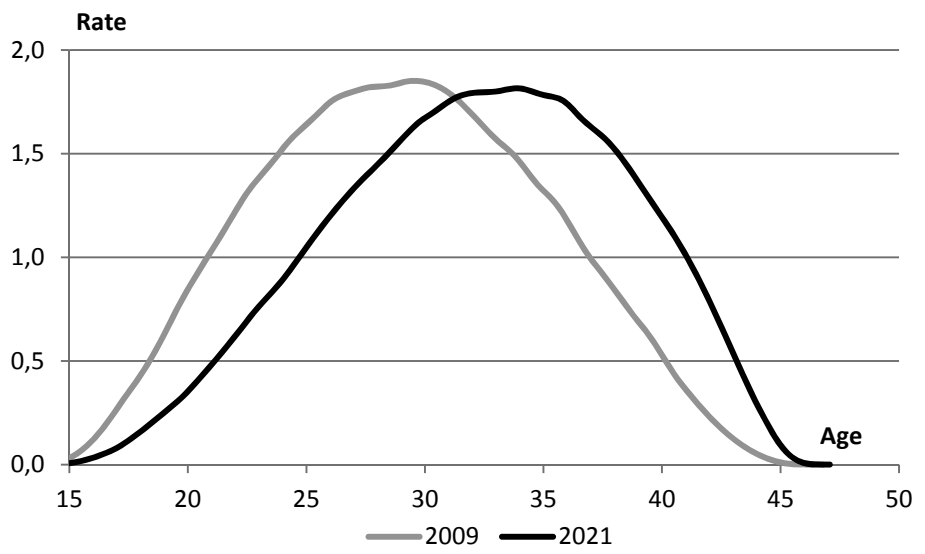

Figure 7. Age-specific fertility rate, 2009, 2021.

It shows that the average age of mothers will probably become even higher and, in 2021, it might reach 32 years.

In the following period, the age of mothers raises, but the shape of the curve remains comparable to that of the previous period. Similarly to other European countries, the average age of mothers is growing in Estonia, too. At present, the average age of the mother at childbirth is 29.9 years, which is by no means the highest in Europe. The average age of mothers in Europe is 30.5 years; the oldest mothers are in Austria and Italy where the average age of mothers is 31.8 and 31.7, respectively.

\section{REFERENCES}

1. Chandola T., Coleman D.A., Hirons R.W. (1999). Resent European fertility patterns: fitting curves to "distorted" distributions. Population Sudies, 53, 317-329.

2. Gilje E. (1969). Fitting curves to age-specific fertility rates: Some examples. Statistical Review of National Central Burleau of Statistics of Sweden, 7, 118-134.

3. Hoem J.M., Madsen D., Nielsen J.L., Ohlsen E.-M., Hansen H.O., Rennermalm B. (1981). Experiments in modelling recent Danish fertility curves. Demography, 18 (2), 231-244.

4. Peristera P., Kostaki A. (2008). Modeling fertility in modern populations. Demographic Research, 16 (6), 141-194. 
5. Rueda-Sabater C., Alvarez-Esteban P.C. (2008). The analysis of age-specific fertility patterns via logistic models. Journal of Applied Statistics, 35 (9), $1055-1070$.

Address for correspondence:

Mare Vähi

Institute of Mathematics and Statistics

Faculty of Science and Technology

University of Tartu, Tartu, Estonia

J. Liivi 2-516, 50409 Tartu, Estonia

E-mail: mare.vahi@ut.ee 